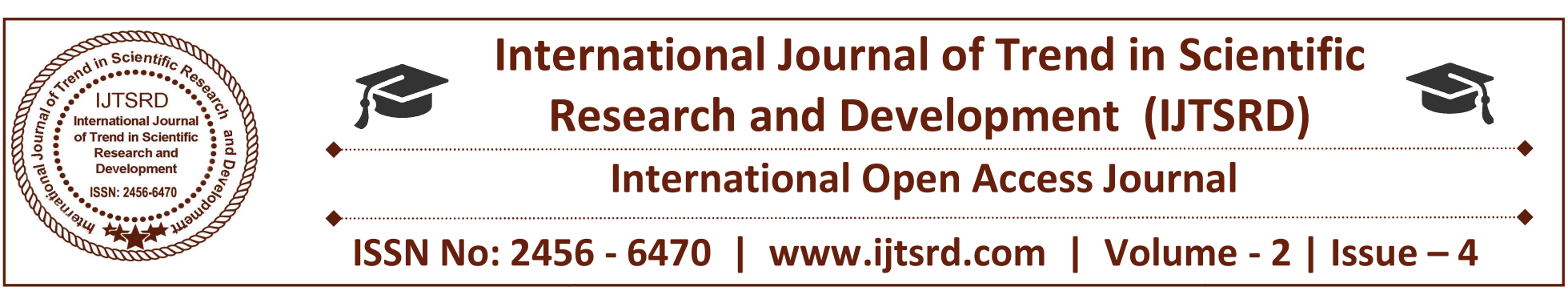

\title{
Analysis and Comparative Study of Image Restoration by using Matlab
}

\author{
Vaishali Kumari, Ranjan Kumar Singh \\ Electronics \& Communication Engineering, Shri Ram College of Engineering \& Management, \\ Palwal, Haryana, India
}

\begin{abstract}
The Image restoration is the recovery of an image that has been degraded by blur and noise. Degradation typically involves blurring of the original image and corruption noise. The recovery of an original image from degraded observations is of paramount importance and can find its application in several scientific areas including medical and diagnostics, authentication automated industry inspection, astronomical imaging and many areas. Image restoration assures good sights of image when it is subjected to further techniques of image processing.
\end{abstract}

In this the presentation of results and comparisons of the restoration algorithms namely Weiner filter, the regularized filter and Lucy Richardson as implemented in MATLAB. The image which will be used that will be degraded by motion blur with Gaussian, Salt and pepper and speckle noise models respectively and restoration of same image using various algorithms mentioned above. The algorithm of image restoration, distinguish themselves from enhance method in that they are based on models for degrading process and for the ideal image.

\section{Objectives:}

- To find out a suitable highly accurate restoration filter to filter and remove. The degradation on an image using Matlab.

- To investigate the strength and limitations of each image restoration filter.

- To find a suitable filter with high performance of filtering and doing a great job of restoring the image.
- To generate an estimate of the original image prior to the degradation.

\section{INTRODUCTION}

Image restoration is a process of restoring image which is degraded by noise or disturbances. Prior knowledge of noise is used to recreate image by removing the ambiguities by applying the inverse process to recreate the original image. It can be done in two domains: spatial domain and frequency domain.

\subsection{A Model of image degradation and restoration process}

An image can be considered as two dimensional signals. In which intensity versus spatial coordinates is plotted in 2-D. The basic unit of an image is called a pixel. The image can be divided into very small blocks called pixels. The 2-D function for image can be given as:

$$
I=f(x, y)
$$

Where $\mathrm{x}$ and $\mathrm{y}$ are spatial coordinates. $(x, y)$ represents a pixel. $I$ is the intensity or grey level value of that spatial coordinate indicated by $(\mathrm{x}, \mathrm{y})$. Which may be defined as amplitude of $f$ at any point $(x, y)$. If the values of the coordinates (spatial coordinates) and the amplitude are finite and discrete, then the image is called digital image. The degraded image $g(x, y)$ can be represented

$$
g(x, y)=h(x, y) * f(x, y)+\eta(x, y)
$$

Where $h(x, y)$ is the degradation function, $f(x, y)$ is the original image, the symbol $*$ indicates

Convolution and $\eta(x, y)$ is the additive noise. 


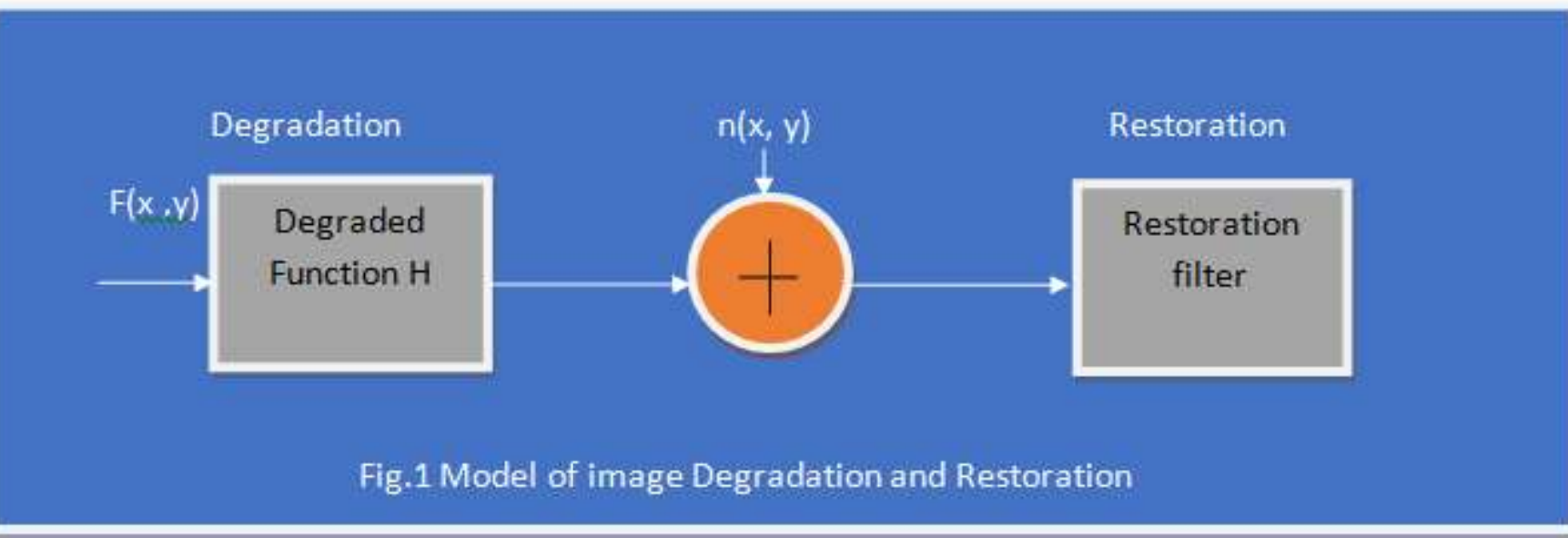

So in the frequency domain we can represent it as

$G(u, v)=F(u, v) H(u, v)+N(u, v)$

\section{TYPES OF NOISE}

\subsection{Gaussian noise}

Gaussian noise is statistical noise having a probability density function (PDF) equal to that of the normal distribution, which is also known as the Gaussian distribution. It can also be interpenetrated as the values that the noise that follows the Gaussiandistributed.

$$
p_{G}(z)=\frac{1}{\sigma \sqrt{2 \pi}} e^{-\frac{(z-\mu)^{2}}{2 \sigma^{2}}}
$$

There is a special case known as white Gaussian noise, in which the values at any pair of times are identically distributed and statistically independent. In testing and modeling, Gaussian noise is used as additive white noise to generate AWGN.

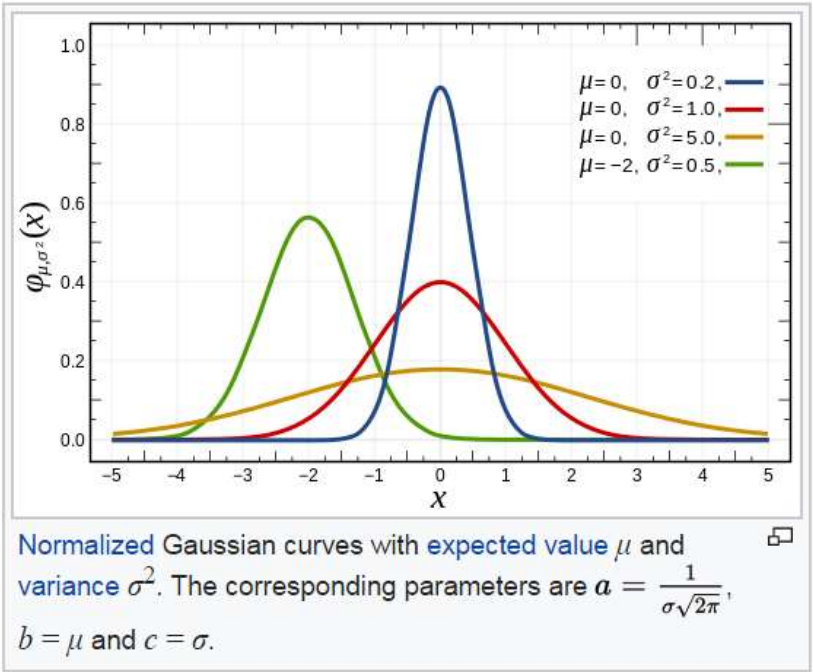

Figure 3.5 Gaussian noise (PDF)
In telecommunications and computer

networking, communication channels can be affected by wideband Gaussian noise coming from many natural sources, such as the thermal vibrations of atoms in conductors, shot noise, black body radiation from the earth and other warm objects, and from celestial sources such as the Sun.

\subsubsection{Gaussian noise in digital images}

Principal sources of Gaussian noise in digital images arise during acquisition e.g. sensor noise caused by poor illumination and/or high temperature, and/or transmission e.g. electronic circuit noise. In digital image processing Gaussian noise can be reduced using a spatial filter, though when smoothing an image, an undesirable outcome may result in the blurring of fine-scaled image edges and details because they also correspond to blocked high frequencies. Conventional spatial filtering techniques for noise removal include: mean (convolution) filtering, median filtering and Gaussian smoothing.

\subsection{Rayleigh Noise}

In probability theory and statistics, the Rayleigh distribution is a continuous probability distribution for positive-valued random variables. A Rayleigh distribution is often observed when the overall magnitude of a vector is related to its directional components. One example where the Rayleigh distribution naturally arises is when wind velocity is analyzed into its orthogonal 2-dimensional vector components. Assuming that each component is uncorrelated, normally distributed with equal variance, and zero mean, then the overall wind speed (vector magnitude) will be characterized by a Rayleigh distribution. Equation define Rayleigh Noise is given by 


$$
P(z)=\left\{\begin{array}{ll}
\frac{2}{b}(z-a) e^{-\left((z-a)^{2}\right) / b} \\
0 \quad \text { for } z \leq a
\end{array} \text { for } z \geq a\right.
$$

A second example of the distribution arises in the case of random complex numbers whose real and imaginary components are independently and identically distributed Gaussian with equal variance and zero mean. In that case, the absolute value of the complex number is Rayleigh-distributed.

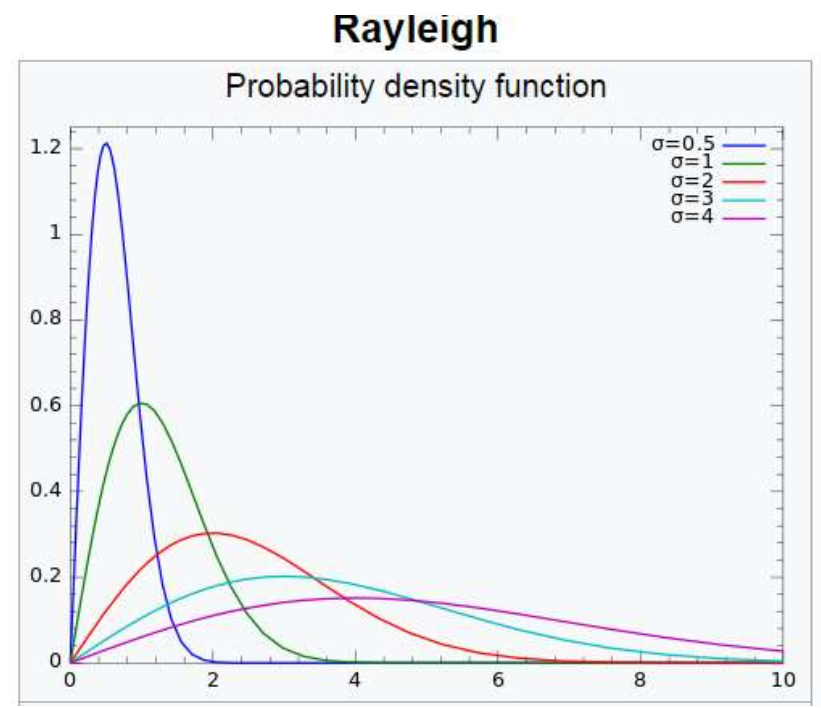

Figure 3.6

\subsection{Uniform Noise}

It is another commonly found image noise i.e. uniform noise. Here the noise can take on values in an interval $[a, b]$ with uniform probability. The PDF is given by

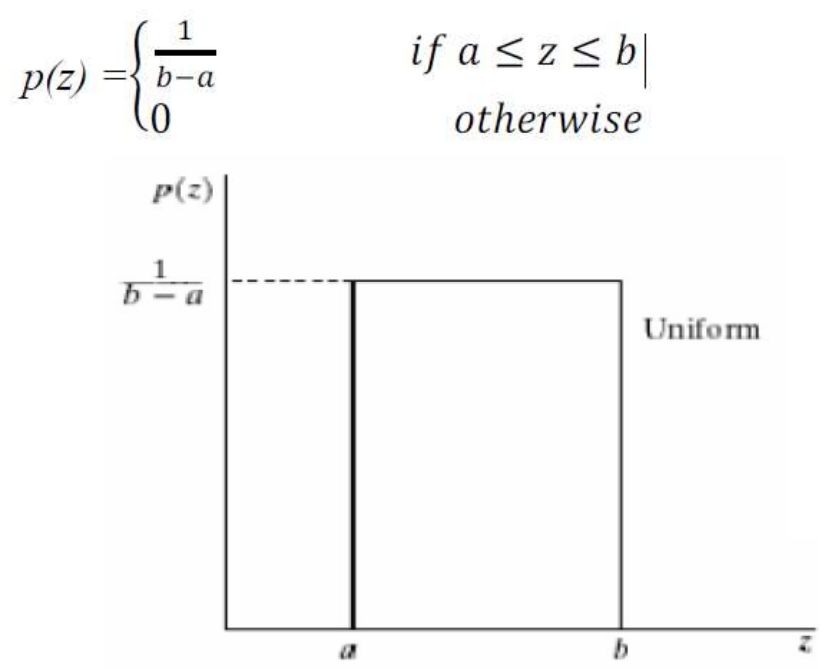

Figure 3.7 Uniform Noise

\subsection{Impulse (salt-and-pepper) Noise}

The PDF of impulse noise is given by

$$
p(z)= \begin{cases}P a & \text { for } z=a \\ P b & \text { for } z=b \\ 0 & \text { otherwise }\end{cases}
$$

If $b>a$, intensity $b$ will appear as a light dot in the image. And the vice-versa, level $a$ will appear like a dark dot.

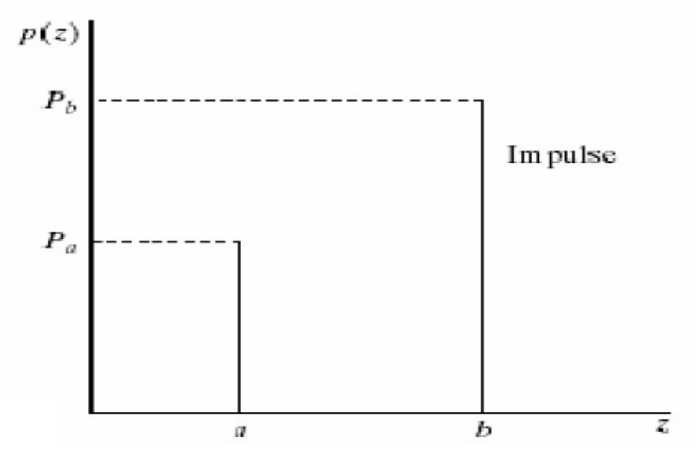

Figure 3.8 Impulse (salt-and-pepper) Noise

If $P a$ or $P b$ is zero, the impulse noise is called unipolar.

\subsection{Exponential Noise}

The PDF of exponential noise is given by

$$
p(\mathrm{z})=\left\{\begin{array}{l}
a e^{-a z} \\
0 \quad \text { for } z<0
\end{array} \text { for } z \geq 0\right.
$$

Where $\mathrm{a}>0$

Mean $=\quad z=1 / a$

Variance $=0=1 / \mathrm{a}^{2}$

\section{FILTERING}

Filtering is the process of removing noise from a noisy image. Filtering is done in two domain: spatial domain and frequency domain.

3.1 FILTERING IN SPATIAL DOMAIN: In spatial domain filtering is done by grazing across coordinate which is in its spatial domain of the image. The most widely used filtering techniques in Image Processing are the Spatial Domain filtering techniques. Spatial Domain is referred as the grid of pixels that represent an image. The relative positions and the values of a local neighborhood of pixels are 
the factors that are used in Spatial Domain filtering technique.

The main idea behind Spatial Domain Filtering is to convolve a mask with the image. The convolution integral of two functions $f(x)$ and $g(x)$ is defined as:

$$
f(x) * g(x)=\int_{-\infty}^{\infty} f(u) g(x-u) d u
$$

Spatial domain filtering can be done in following ways.

\subsection{Mean filters}

Arithmetic mean filter- In this type of mean filter the middle pixel value of the mask is replaced with the arithmetic mean of all the pixel values within the filter window. A mean filter simply smoothes local variations in an image. Noise is reduced and as a result the image smoothens, but edges within the image get blurred.

Geometric mean filter- The working of a geometric mean filter is same as the arithmetic mean filter; the only difference is that instead of taking the arithmetic mean the geometric mean is taken.

- Harmonic mean filter- In the harmonic mean method, the gray value of each pixel is replaced with the harmonic mean of gray values of the pixels in a surrounding region.

- Log filter-Log Filtering includes sequential processing of soothing for noise remove immediately and computing second derivative.

\subsection{Order statistic filters}

Max and Min filter- The maximum filter is defined as the maximum of all pixels within a local region of an image. So it replaces the center pixel value with the maximum value of pixel in the subimage window. Similarly the minimum filter is defined as the minimum of all pixels within a local region of an image and the center pixel value is replaced with the minimum value of pixel in the subimage window.
- Midpoint filter- Order-statistics filters are based on ordering the pixels contained in the mask. Median filter comes under this class of filters. Median filter replaces the value of a pixel with the median value of the gray levels within the filter window or mask.

\subsection{FREQUENCY DOMAIN FILTERING}

In frequency domain filtering is done by converting image into frequency domain transform. The frequency domain is an alternate way to represent an image. It deals with the frequency of the gray levels of the pixels in the image i.e. the variation in the gray level. Considering the frequency components of an image can provide an insight and rationale for certain filtering and processing operations. In frequency domain filtering the image is mapped from spatial domain to frequency domain by taking Fourier transform of the image. After mapping filtering operation done on the image (like low pass and high pass filtering etc). After doing the filtering operation the image is remapped to spatial domain by inverse Fourier transform to obtain the restored image.

Filter based on frequency domain filtering are as follows.

- Low pass filters

- High pass filters

- Weiner filtering- Wiener filtering incorporates both the degradation function and statistical characteristics of noise into the restoration process. This method is found on considering images and noise as random variables, and the objective is to find an estimate $f$ of the uncorrupted image $f$ such that the mean square error between then is minimized.

$$
e^{2}=E\left\{(f-\hat{f})^{2}\right\}
$$

In the frequency domain $f^{\wedge}$ is given by

$\left.\hat{F}(u, v)=\left[H^{*}(u, v) S_{f}(u, v)\right)\left\{S_{f}(u, v)|H(u, v)|^{2}+S_{n}(u, v)\right\}\right] G(u, v)$ 
International Journal of Trend in Scientific Research and Development (IJTSRD) ISSN: 2456-6470

\begin{tabular}{|l|l|l|l|l|}
\hline & Salt \& pepper & Speckle & Gaussian & Poisson \\
\hline s Arithmetic mean filter & 71.2881 & 72.3291 & 67.2821 & 76.3676 \\
\hline Geometric mean filter & 61.6017 & 69.2991 & 67.2582 & 75.5991 \\
\hline Harmonic mean filter & 54.5830 & 54.5672 & 54.5495 & 54.5762 \\
\hline Min filter & 54.5683 & 54.5550 & 54.5785 & 54.5722 \\
\hline Max filter & 54.5876 & 54.5916 & 54.6008 & 54.5843 \\
\hline Median filter & 76.9848 & 69.5576 & 67.0526 & 76.5405 \\
\hline Wiener filtering & 67.6746 & 70.5397 & 67.2539 & 79.1092 \\
\hline
\end{tabular}

TABLE.7.1 List of PSNR for different Noise and different filter with same noise percentage.

\section{CONCLUSIONS AND FUTURE WORK}

In this thesis from reference to given Table 7.1 and Graph 7.1, Arithmetic mean filter is preferred for Salt \& Pepper and speckle noise where as geometric mean filter is best for Gaussian and Poisson both these filter smoothen the image and loose less detail. Harmonic filter works best with salt noise only. Median filters are effective in presence of unipolar and bipolar impulse noise. Max filter is useful for finding brightest points and thus reduces pepper noise. Min filter is useful for finding darkest points. Wienier filter works best on Poisson noise. The work done is only on gray scale images. It can be extended to colored images as well TEM images. By extracting RGB plan individually and applying filtration. Image restoration mainly required prior knowledge of the degradation function. As well as Optimal method can be used by checking PSNR of image while coding.

\section{References}

1) Siba Prasad Tudu, A Bharath Kumar Reddy; IMAGE RESTORATION TECHNIQUE , NIT ROURKELA, MAY 2013

2) Introduction to MATLAB referred link is "http://in.mathworks.com/help/images/getting started-with-image-processingtoolbox.html'".

3) Admore Gota, Zhang Jian Min; Analysis and Comparison on Image Restoration Algorithms Using MATLAB, Tianjin University of Technology and Education (TUTE), International Journal of Engineering Research \& Technology (IJERT)Vol. 2 Issue 12, December - 2013

4) A. Kumar and F. Shaik, Image Processing in Diabetic Related Causes, Forensic and Medical Bioinformatic, DOI 10.1007/978-981-287-624-9, 2016.

5) R. C. Gonzalez and R. E. Woods, Digital Image Processing, 2nd ed. Prentice-Hall India, 2005. 